\title{
Cobalt-Doped ZnO Nanorods Coated with Nanoscale Metal-Organic Framework Shells for Water-Splitting Photoanodes
}

\author{
Alejandro Galán-González,* Aswathi K. Sivan, Javier Hernández-Ferrer, Leon Bowen, Lorenzo Di Mario, \\ Faustino Martelli, Ana M. Benito, Wolfgang K. Maser, Mujeeb Ullah Chaudhry, Andrew Gallant, \\ Dagou A. Zeze, and Del Atkinson*
}

Cite This: ACS Appl. Nano Mater. 2020, 3, 7781-7788

Read Online

\section{ACCESS \\ W Metrics \& More \\ Article Recommendations \\ Supporting Information}

ABSTRACT: Developing highly efficient and stable photoelectrochemical (PEC) water-splitting electrodes via inexpensive, liquid phase processing is one of the key challenges for the conversion of solar energy into hydrogen for sustainable energy production. $\mathrm{ZnO}$ represents one the most suitable semiconductor metal oxide alternatives because of its high electron mobility, abundance, and low cost, although its performance is limited by its lack of absorption in the visible spectrum and reduced charge separation and charge transfer efficiency. Here, we present a solution-processed watersplitting photoanode based on Co-doped $\mathrm{ZnO}$ nanorods (NRs) coated with a transparent functionalizing metal-organic framework (MOF). The light absorption of the $\mathrm{ZnO}$ NRs is engineered toward

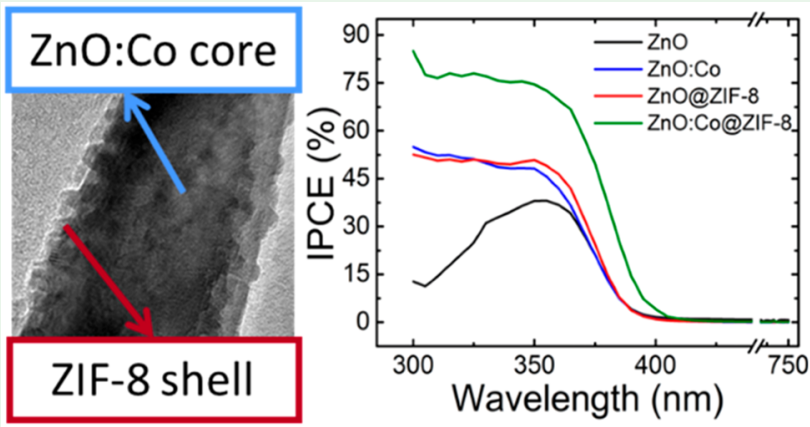
the visible region by Co-doping, while the MOF significantly improves the stability and charge separation and transfer properties of the NRs. This synergetic combination of doping and nanoscale surface functionalization boosts the current density and functional lifetime of the photoanodes while achieving an unprecedented incident photon to current efficiency (IPCE) of $75 \%$ at $350 \mathrm{~nm}$, which is over 2 times that of pristine $\mathrm{ZnO}$. A theoretical model and band structure for the core-shell nanostructure is provided, highlighting how this nanomaterial combination provides an attractive pathway for the design of robust and highly efficient semiconductor-based photoanodes that can be translated to other semiconducting oxide systems.

KEYWORDS: nanorod, zinc oxide, water splitting, metal-organic framework, photoelectrochemical, doping

\section{INTRODUCTION}

The development of efficient, robust, and cost-effective watersplitting cells ${ }^{1-7}$ is key to establishing sustainable hydrogen production as a renewable energy source..$^{8-11} \mathrm{~A}$ viable avenue for this production is photoelectrochemical water splitting, which requires suitable photoelectrodes that can efficiently supply significant current densities over a long lifetime. This requires engineering semiconducting materials to have a suitable bandgap and band structure that enhance light absorption, charge separation, and transport. Metal oxide semiconductors such as titanium dioxide, ${ }^{12}$ hematite, ${ }^{13}$ zinc oxide, ${ }^{14-16}$ and perovskites ${ }^{17}$ have been widely employed as photoanodes in PEC water splitting.

Among these, $\mathrm{ZnO}^{18}$ is an attractive candidate for watersplitting photoanodes due to its high electron mobility, natural abundance, and low cost. Importantly, nanotechnological approaches using $\mathrm{ZnO} \mathrm{NR}$ arrays have proven to be an effective alternative route to the development of efficient photoanode compared to thin-film-based ones because of the vastly increased surface area and shorter lateral carrier transfer length. However, unprotected $\mathrm{ZnO} \mathrm{NRs}$ suffer from low photogenerated current density due to charge recombination prompted by surface states, subpar light absorption due to a wide bandgap, average efficiency, and a poor functional lifetime due to photocorrosion, i.e., the irreversible physical and chemical changes induced by oxidation and reduction processes of the photogenerated charge carriers. There have been significant efforts to overcome the characteristic shortcomings of $\mathrm{ZnO}$ to exploit its potential benefits, with strategies such as doping ${ }^{19}$ or NR decoration, ${ }^{20}$ with either quantum dots or carbon-based materials, being extensively explored.

Two distinct strands of current $\mathrm{ZnO}$ research present complementary opportunities to engineer the performance of $\mathrm{ZnO} \mathrm{NRs}$ as a photoanode. First, doping of $\mathrm{ZnO}$ with elements such as cobalt or nitrogen, by using low-cost processes, ${ }^{21,22}$ has been successfully demonstrated as a viable

Received: $\quad$ May 14, 2020

Accepted: July 13, 2020

Published: July 15, 2020 
bandgap engineering process. Here, cobalt is integrated in the form of a substitutional dopant in the wurtzite $\mathrm{ZnO}$ lattice, with the $\mathrm{Co}^{2+}$ ions occupying the position of $\mathrm{Zn}^{2+}$ ions. ${ }^{23}$

Second, the nanotechnological integration of $\mathrm{MOFs}^{24}$ with $\mathrm{ZnO}$. MOFs are a class of organometallic nanomaterials characterized by their organic moieties, large surface areas, and chemical compatibility. ${ }^{25}$ Among the many MOF families, the zeolitic imidazolate frameworks are one of the most studied because of their chemical and mechanical properties. ${ }^{26,27}$ One of the most studied materials within this family is ZIF-8, which is formed by $\mathrm{Zn}$ nuclei tetrahedrally coordinated by 2methylimidazole organic linkers. ${ }^{28}$ The integration of a ZIF-8 shell around $\mathrm{ZnO}$ cores has shown potential stability, charge generation, and charge transfer improvements. ${ }^{29,30}$ One example is the growth of the ZIF-8 as a shell around $\mathrm{ZnO}$, which is typically achieved by hydrothermal growth in a methanol or a water/dimethylformamide solution containing a zinc source and the organic linker, which is easily incorporated with $\mathrm{ZnO} \mathrm{NRs}$ by the solvothermal conversion of $\mathrm{ZnO}$ into ZIF-8. ${ }^{30,31}$ Even though this integration has been reported in the literature, the interplay between the ZIF-8 shell and $\mathrm{ZnO}$ core has not been adequately addressed so far since there remains no conclusive mechanistic model that explains the charge transfer between the core and shell at this time.

To date, these two strands have been independently researched as different pathways to enhance the performance of $\mathrm{ZnO}$ as a photoanode. In this work, these two research strands are successfully brought together for the first time to create a new class of functionally doped $\mathrm{ZnO} \mathrm{NRs} / \mathrm{MOF}$ nanomaterials. We demonstrate that an unprecedented incident to photon current efficiency (IPCE-75\%) can be achieved in the near-visible region (at $350 \mathrm{~nm}$ ) using these materials unlike traditional approaches that operate only in the UV region. In addition, a functional model for the synergetic behavior of the combination of these two nanomaterials is given to provide a theoretical framework to consider how their interplay leads to the enhanced functional performance observed.

\section{RESULTS AND DISCUSSION}

The $\mathrm{ZnO}$ :Co NRs are grown by a two-step process consisting of the spin-coating of a $\mathrm{ZnO}$ seed layer and subsequent NR growth with the well-established chemical bath deposition ${ }^{32,33}$ (CBD). Cobalt is introduced into the growth solution, where it is incorporated into the $\mathrm{ZnO}$ lattice, substituting $\mathrm{Zn}$ atoms during growth through competitive reactions, as shown by the $\mathrm{X}$-ray diffraction and X-ray photoelectron spectroscopy (XPS) results in Figures S1 and S2 of the Supporting Information. A $1 \% \mathrm{Co}: \mathrm{Zn}$ ratio in solution is discussed here. This value is only the nominal value; the actual value of the amount of cobalt integrated in the $\mathrm{ZnO} \mathrm{NRs}$ is expected to be considerably lower. $^{23}$ Other cobalt doping levels were considered and studied, with $1 \%$ yielding the best performance and therefore being the focus of this work. The ZIF-8 shell is grown efficiently by a previously unreported spin-coating route based on the deposition of a methanol solution containing the imidazole linker onto the $\mathrm{ZnO}$ NRs, followed by surface conversion of the $\mathrm{ZnO}$ NRs. The various steps of the growth of the core-shell NRs and the structure of the photoanode in the photoelectrochemical cell are illustrated in Figure 1. This unexplored MOF deposition processing creates a fast, facile, and controllable route for the growth of nanoscale ZIF-8 shells on $\mathrm{ZnO}$ NRs. Four different classes of samples are discussed

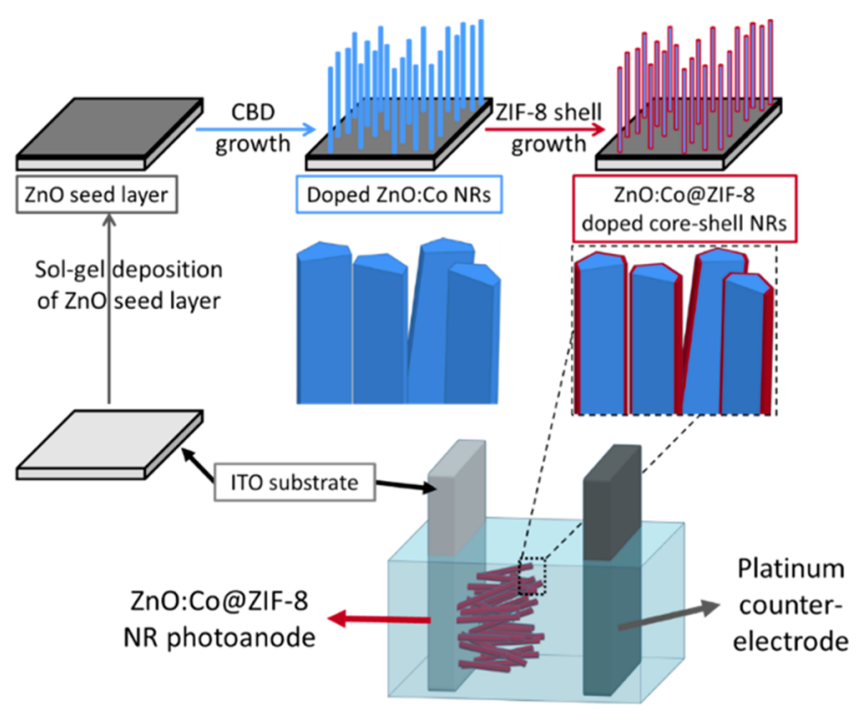

Photoelectrochemical cell

Figure 1. Schematic illustration of the ZnO:Co@ZIF-8 core-shell NRs growth, with the three consecutive steps that form the seed layer deposition, NR growth, and ZIF-8 shell growth. A schematic of the photoelectrochemical cell used for the characterization in this work is included, with its NR-based working electrode and platinum counter electrode labeled.

here and are labeled: $\mathrm{ZnO}, \mathrm{ZnO}: \mathrm{Co}, \mathrm{ZnO} @ Z I F-8$, and ZnO:Co@ZIF-8 representing ZnO NRs, 1\% Co-doped NRs, core-shell NRs, and $1 \%$ Co-doped core-shell NRs, respectively.

These Co-doped ZnO core@ZIF-8 shell NRs are shown to have unprecedented IPCE values (Figure 2a) for reported $\mathrm{ZnO}$ systems because of the synergetic combination of MOF and doping. Scanning electron micrographs (SEM) of $\mathrm{ZnO}$ :Co and ZnO:Co@ZIF-8 are presented in Figures $2 \mathrm{~b}$ and 2c, respectively. The diameter of both $\mathrm{ZnO}: \mathrm{Co}$ and $\mathrm{ZnO}: \mathrm{Co} @$ ZIF-8 NRs is around $75 \mathrm{~nm}$, and the morphological changes introduced by the MOF shell appear as roughened surfaces on the lateral faces of the NRs (Figures S3-S7). Transmission electron microscopy (TEM) demonstrates the presence of the MOF shell around the NR core (Figure 2d). Effectively, the amorphous outer layer on the NRs (outlined by the red dashed lines) is the ZIF- 8 shell, which covers the core with a thickness of about $2 \mathrm{~nm}$ in a conformal way. The crystalline phase of the NRs was determined by high-resolution TEM (HRTEM), as shown in Figure 2g. The texture highlighted in red in this image is a result of the ZIF-8 shell growth (see Figure S3). The lattice fringe spacing was of $0.26 \mathrm{~nm}$, corresponding to the lattice planes of hexagonal wurtzite $\mathrm{ZnO}$. The crystalline phase was corroborated by the indexed selected area electron diffraction pattern, shown in the Figure $2 \mathrm{~g}$ inset, which is typical for wurtzite phase $\mathrm{ZnO}$. This reveals that the shell growth and the introduction of Co into the lattice do not alter the single crystal wurtzite phase of the $\mathrm{ZnO}$ NRs. Energydispersive X-ray spectroscopy (EDX) chemical mapping (Figure 2e,f,h,i) shows that zinc and oxygen are homogeneously distributed across and along the NRs. This equal distribution of concentrations is also observed for Co and $\mathrm{N}$. Nitrogen is present only in the ZIF-8 shell that conformally coats the NR and is thus present over the surface of the NRs. Furthermore, the concentration of Co around the edges is lower compared to the central part of the NR, while the 


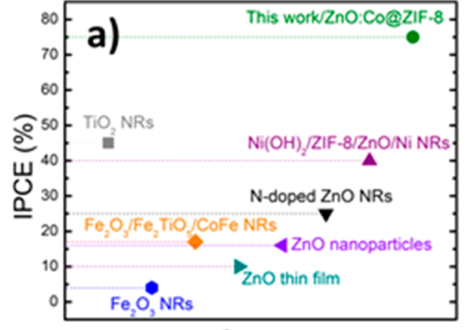

System
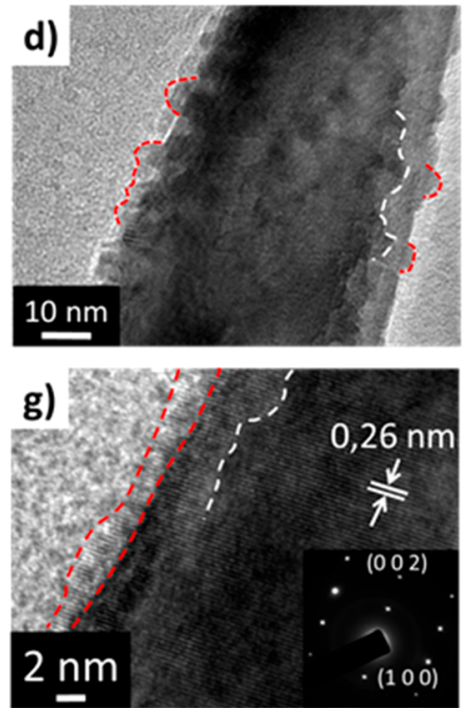
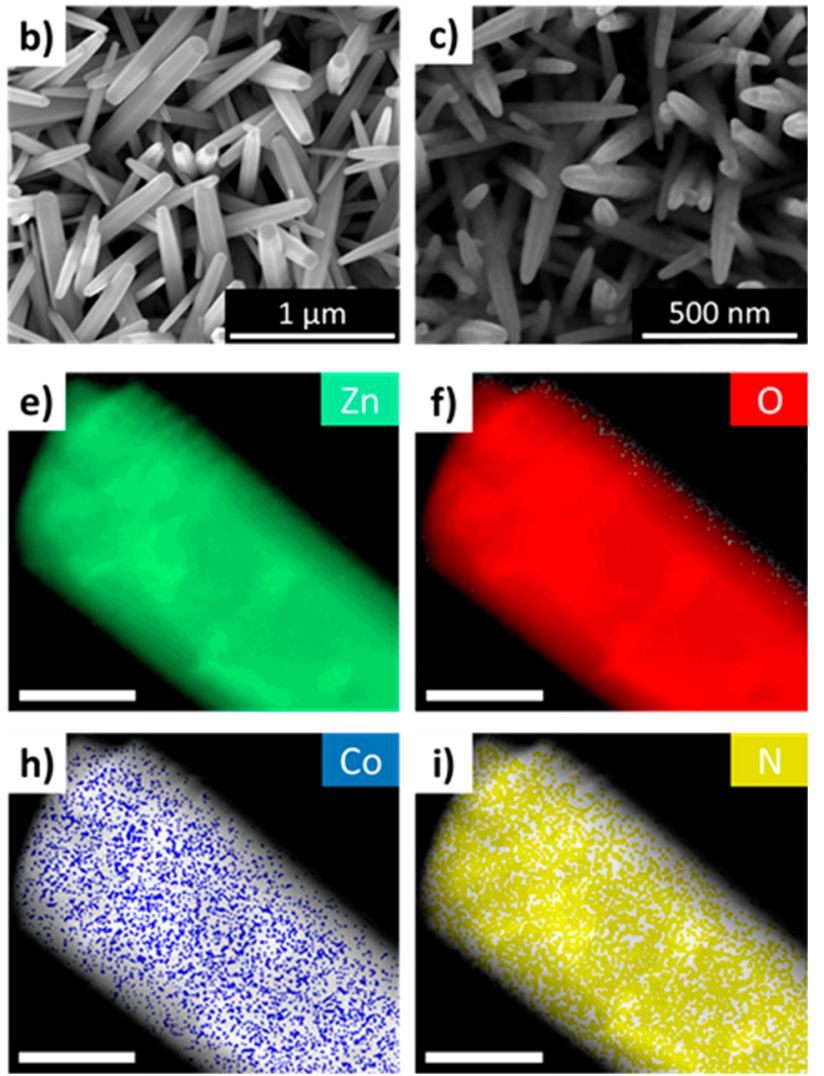

Figure 2. (a) Comparative graph of the water-splitting photoanode performance in terms of IPCE of different sets of representative metal oxide semiconductors used in the literature revealing record IPCE value for ZnO:Co@ZIF-8 used in this work (see Figure 3c). Additional details can be found in Table S1. (b) SEM micrograph of as-grown ZnO:Co NRs. (c) SEM micrograph of the core-shell ZnO:Co@ZIF-8 NRs. (d) TEM image showing the core-shell structure. The red dashed highlights correspond to the sidewall roughness seen in (b), while the white dashed line demonstrates that surface roughness on the NR. There are two clearly distinct contrasting regions on the right side of the NR (between the white and red dashed lines), attributed to different NR diameter in this region, which are not seen for the pristine ZnO NRs. (g) HR-TEM of the same NR. The red dashed lines indicate the presence of the conformal ZIF- 8 shell, while the white line shows the textured surface roughness seen in the surface of the NRs as a result of the shell growth. The inset in $(\mathrm{g})$ presents the diffraction pattern of the NR, indicating the wurtzite single crystal structure. (e, f, h, i) Zinc, oxygen, cobalt, and nitrogen elemental maps of Co-doped core-shell NRs; the scale bars indicate $50 \mathrm{~nm}$.

concentration of $\mathrm{N}$ is similar around the edges and in the inner regions of the NR, which is consistent with a homogeneous coating of the $\mathrm{ZnO}$ :Co core. No quantification of the elemental composition of the core-shell NRs was pursued because of the extremely small quantities of $\mathrm{Co}$ and $\mathrm{N}$ present in them, which would compromise an accurate compositional analysis by EDX. In addition, XPS was also proven not to be accurate enough to detect the Co signal (Figure S2c); therefore, the elemental maps here provided are of qualitative value.

The photoelectrochemical response of this doped NR-MOF system is presented in Figure 3 for the four types of samples. Figure 3a shows the cyclic sweep voltammetry (CSV) of the different samples as photoelectrodes in a $\mathrm{pH} 13$ alkaline solution and in the potential region of -0.51 to $1.33 \mathrm{~V}$ versus the reversible hydrogen electrode (RHE). The CSV was measured at a scan rate of $10 \mathrm{mV} \mathrm{s}^{-1}$ under AM $1.5 \mathrm{G}$ illumination $\left(300 \mathrm{~mW} \mathrm{~cm}{ }^{-2}\right)$. Compared to $\mathrm{ZnO}$, both the ZnO@ZIF-8 and ZnO:Co samples show a 30\% increase in the current density. When both doping and MOF are combined in ZnO:Co@ZIF-8, the current density is almost twice as large as that of the $\mathrm{ZnO} \mathrm{NRs}$, clearly underlining the synergetic benefits of combining cobalt doping and the ZIF-8 shell on the performance of this modified $\mathrm{ZnO}$-based photoanode. Furthermore, the shell provides a protective layer against photocorrosion, which can be seen in the low potential regions $(-0.51$ to $0.3 \mathrm{~V})$. Here, some cathodic peaks indicative of undesired reduction processes also appear and are subsequently eliminated when the NRs are effectively passivated by the ZIF- 8 shell. These peaks are related to the formation of stable intermediates (peroxides and superoxides) during the oxidation of water. The formation of these stable peroxides on the surface of the $\mathrm{ZnO} N R$ indicates that the water in the interphase is not fully oxidized. ${ }^{34}$ When the ZIF-8 shell is incorporated on the NRs, these cathodic peaks are no longer present, signaling the disappearance of the stable intermediate oxides, hence the improvement of the current density for the core-shell nanostructure. The electronic response to light exposure of the photoanodes is probed in transient amperometric studies using chopped AM 1.5 G illumination at $1.33 \mathrm{~V}$ vs RHE (see Figure 3b). All samples show a characteristic on/off behavior and good stability. $\mathrm{ZnO}$ and $\mathrm{ZnO}$ :Co show an upward trend for the first few cycles before stabilizing at their corresponding current densities, attributed to the formation of the previously mentioned oxide intermediates. A small transitionary state appears for the core-shell samples, which promptly stabilizes. The electronic response to light across the visible spectrum is critical to demonstrating and understanding the performance benefits obtained from these nanoengineered materials. Wavelength- 
a)

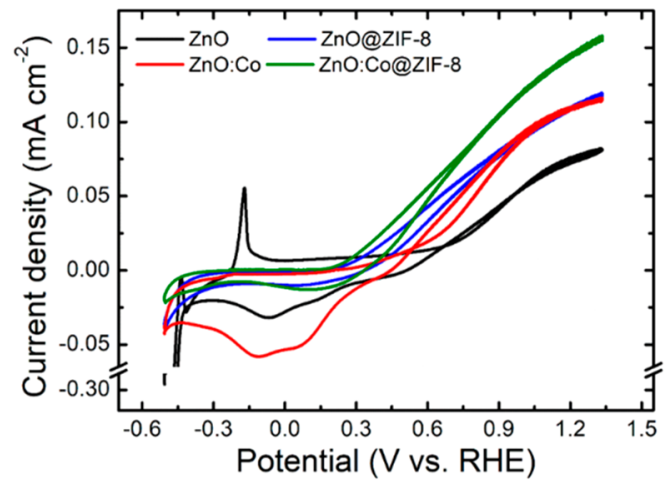

c)

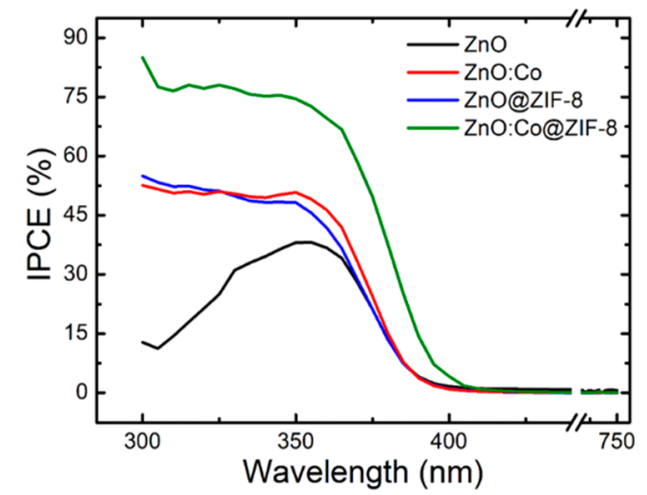

b)

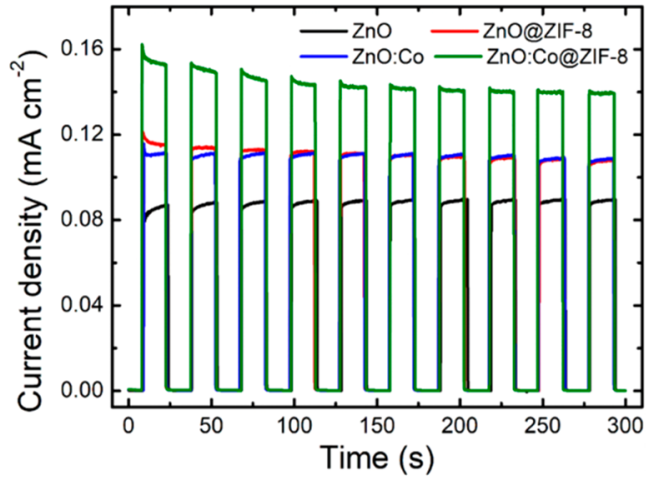

d)

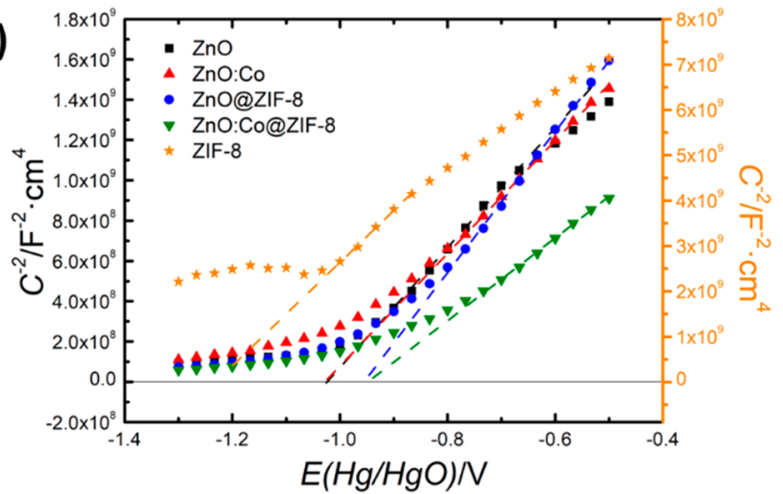

Figure 3. (a) Cyclic sweeping voltammetry under AM $1.5 \mathrm{G}$ illumination for the $\mathrm{ZnO}$ NR photoanodes. For voltammetry in dark conditions, see Figure S8. (b) Transient amperometric studies performed at $1.33 \mathrm{~V}_{\mathrm{RHE}}$ under chopped AM $1.5 \mathrm{G}$ illumination. The small differences in the time scales are due to manual light chopping. (c) IPCE measurements at $1.33 \mathrm{~V}_{\mathrm{RHE}}$. (d) Impedance measurements taken at frequencies from 105 to 0.1 $\mathrm{Hz}$ in the potential range of -0.5 to $-1.3 \mathrm{~V}$ vs $\mathrm{Hg} / \mathrm{HgO}$ electrode, with the corresponding equivalent circuit shown in Figure S10. The dashed lines indicate the linear best fits, the intercepts of which indicate the relative positions of the conduction band.

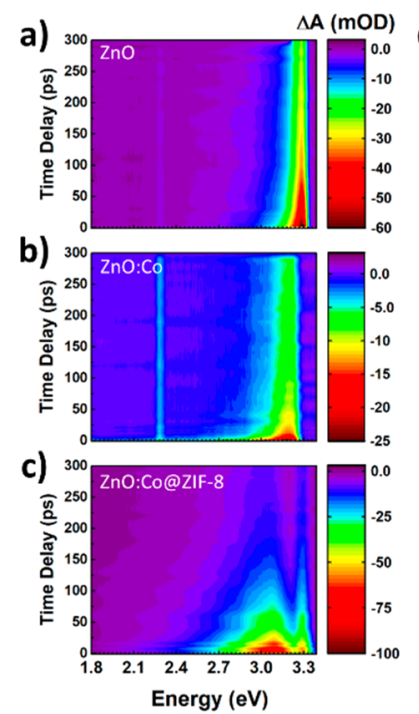

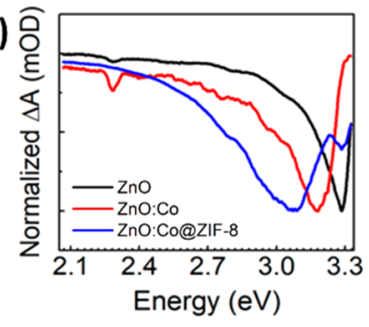

g)

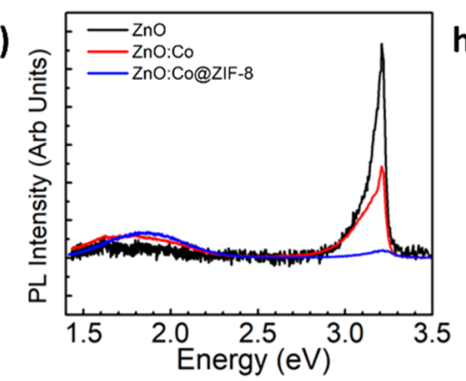

e)

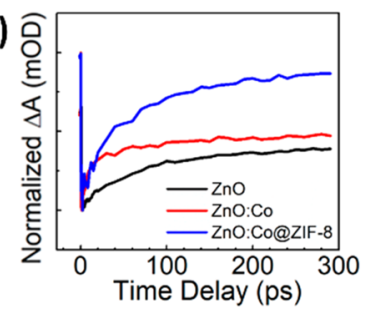

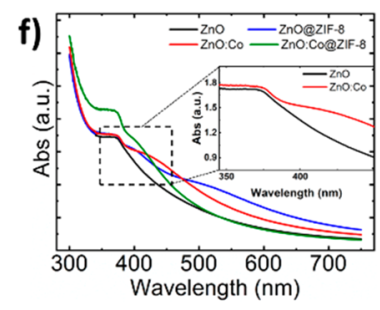

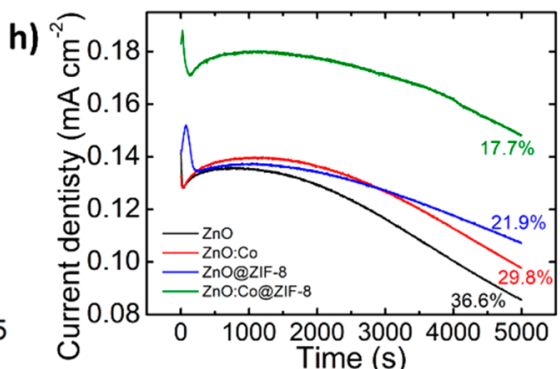

Figure 4. Two-dimensional false color map of the transient absorption spectra of (a) ZnO, (b) ZnO:Co, and (c) ZnO:Co@ZIF-8 measured at room temperature as a function of the probe energy ( $x$-axis) and the delay time between pump and probe ( $y$-axis). The excitation intensity was 260 $\mu \mathrm{J} \mathrm{cm}^{-2}$. The signal at $2.25 \mathrm{eV}(550 \mathrm{~nm})$ corresponds to the second order of the pump $(275 \mathrm{~nm})$ passing through the spectrometer. (d) Energy dependence of the bandgap bleaching at a delay of 1 ps for $\mathrm{ZnO}, \mathrm{ZnO}: \mathrm{Co}$, and $\mathrm{ZnO}$ :Co@ZIF-8. (e) Time dependence of the bandgap bleaching for $\mathrm{ZnO}, \mathrm{ZnO}: \mathrm{Co}$, and $\mathrm{ZnO}: \mathrm{Co} @ Z I F-8$. (f) UV-vis spectroscopy of the four samples studied. The inset shows the shoulder related to the strong $\mathrm{sp}-\mathrm{d}$ interaction resulting of the substitution of $\mathrm{Zn}^{2+}$ with $\mathrm{Co}^{2+}$. (g) Photoluminescence spectra of $\mathrm{ZnO}, \mathrm{ZnO}: \mathrm{Co}$, and $\mathrm{ZnO}: \mathrm{Co} @ \mathrm{ZIF}-8$. (h) Stability measurements showing the enhanced lifetime of the core-shell photoanodes. The numbers indicate the total current density lost at the end of the measurement with respect to the stable point at $1000 \mathrm{~s}$ for each photoanode.

dependent IPCE measurements at $1.33 \mathrm{~V}$ vs RHE are shown in Figure 3c. Compared to the $\mathrm{ZnO}$, both $\mathrm{ZnO@ZIF-8} \mathrm{and}$ $\mathrm{ZnO}$ :Co show a large efficiency improvement, increasing by
$10 \%$ and $13 \%$, respectively, at $350 \mathrm{~nm}$, which corresponds to the position of the highest IPCE of $\mathrm{ZnO}$. Furthermore, the separate additions of doping and MOF increase the efficiency 
for wavelengths in the UV region, rising to $42 \%$ and $40 \%$ at $300 \mathrm{~nm}$ for the $\mathrm{ZnO} @ \mathrm{ZIF}-8$ and $\mathrm{ZnO}$ :Co, respectively. No significant red-shift in the IPCE was observed for ZnO@ZIF-8, while a small red-shift could be observed for $\mathrm{ZnO}$ :Co compared to pristine $\mathrm{ZnO}$. Most significantly, the combination of doping and MOF in ZnO:Co@ZIF-8 demonstrates significant performance improvements with the IPCE increasing to $75 \%$ at $350 \mathrm{~nm}$ and a remarkable $85 \%$ at $300 \mathrm{~nm}$, while showing a clear red-shift of $15 \mathrm{~nm}$ for the onset. MottSchottky plots (Figure 3d) were obtained by electrochemical impedance spectroscopy to assess the relative positions of the conduction band of the photoanodes, as indicated by the intercepts of the linear best fitting lines shown. Co doping does not alter the position of the conduction band, having a value of $-1.03 \mathrm{~V}$, which is almost the same as that of $\mathrm{ZnO}$. However, the introduction of the ZIF- 8 shell shifts the conduction band to a lower potential of $-0.95 \mathrm{~V}$ for both undoped and doped NRs. Additionally, for ZnO:Co@ZIF-8 electrodes, the slope of Mott-Schottky plots decreases, indicating an increase of the charge carrier concentration in the $\mathrm{ZnO}$. This reveals a strong interaction between the ZIF- 8 shell and the $\mathrm{ZnO}$ :Co core, resulting in an effective electron transfer from the shell to the core that which is facilitated by the presence of additional energy levels in the bandgap introduced by the cobalt doping.

Additional optical and electrochemical analyses were performed (Figure 4) to give further insight into the separate influences of the cobalt doping and the ZIF- 8 shell. Fasttransient absorption spectroscopy (FTAS) analysis, measured by a pump-probe method, is shown for $\mathrm{ZnO}, \mathrm{ZnO}: \mathrm{Co}$, and ZnO:Co@ZIF-8 in Figure 4a-c. The pump energy was 4.51 $\mathrm{eV}(275 \mathrm{~nm})$, and the broad supercontinuum probe was in the $\mathrm{UV}-$ vis region. The $\mathrm{ZnO}$ NRs show a narrow, negative signal, indicative of an absorption bleaching centered at $3.3 \mathrm{eV}$, corresponding to the bandgap of $\mathrm{ZnO}$ (Figure $4 \mathrm{a}$ ). Cobalt doping (Figure $4 \mathrm{~b}$ ) induces a red-shift of the bandgap to lower energies, with the absorption bleaching signal centered at 3.18 $\mathrm{eV}$ that equates to a wavelength shift of $15 \mathrm{~nm}$. Considering ZnO:Co@ZIF-8 (Figure 4c), the bleaching maxima is shifted further to $3.08 \mathrm{eV}$, with a less intense peak at the position of the bandgap of $\mathrm{ZnO}$, which is attributed to the $\mathrm{ZnO}$ seed layer. This absorption bleaching signal is rather wide and serves as explanation for the shift shown in the IPCE measurements of Figure 3c. The normalized difference in absorbance $(\Delta A)$ as a function of probe energy at a delay of 1 ps is shown in Figure $4 \mathrm{~d}$, which clearly illustrates the red-shift in the absorption bleaching for ZnO:Co and ZnO:Co@ZIF-8. Furthermore, the latter shows a strong absorption extending over the entire probe range. The time dependence of the bandgap bleaching obtained from the FTAS is presented in Figure 4e, Here, a faster decay of the bandgap bleaching in the Co-doped NRs and in the core-shell NRs is shown, which is attributed to alternative nonradiative decay pathways due to the introduction of a $\mathrm{d}-\mathrm{d}$ transition in the bandgap by effect of the Codoping. ${ }^{35,36}$ The UV-vis stationary absorption spectra of the photoanodes are provided in Figure 4f. These show a red-shift of the absorption bleaching in the presence of Co doping that can be attributed to strong $\mathrm{sp}-\mathrm{d}$ exchange interaction ${ }^{39}$ (see the inset in Figure 4f). The substitution of $\mathrm{Zn}^{2+}$ by $\mathrm{Co}^{2+}$ leads to a stronger interaction between the sp band electrons and the localized d electrons of Co. For ZnO:Co@ZIF-8, there is an absorption red-shift compared to the other three materials, as demonstrated by the fact that $\mathrm{ZnO}: \mathrm{Co} @ Z I F-8$ achieves the same absorption as $\mathrm{ZnO}, \mathrm{ZnO} @ \mathrm{ZIF}-8$, and $\mathrm{ZnO}$ :Co at a wavelength $15 \mathrm{~nm}$ higher, from 380 to $395 \mathrm{~nm}$. A further small shift is seen at around $405 \mathrm{~nm}$, corresponding to the wide absorption bleaching shown in Figure 4c. This shift in absorption is related to the shift observed in the IPCE and the Mott-Schottky measurements of ZnO:Co@ZIF-8. Furthermore, ZnO:Co@ZIF-8 also shows 30\% larger absolute absorption between 300 and $350 \mathrm{~nm}$ compared to the other photoanodes.

Photoluminescence (PL) spectroscopy was performed on $\mathrm{ZnO}, \mathrm{ZnO}: \mathrm{Co}$, and $\mathrm{ZnO}$ :Co@ZIF-8 to further understand the influence of the Co-doping and the shell on the NR properties (Figure $4 \mathrm{~g}$ ). The $\mathrm{PL}$ of $\mathrm{ZnO}$ shows a strong peak around 3.27 $\mathrm{eV}$ corresponding to the band edge (BE) recombination. For $\mathrm{ZnO}: \mathrm{Co}$, the intensity of the BE PL is significantly reduced, while a broader peak centered around $1.9 \mathrm{eV}$ appears; this broad peak is attributed to the characteristic oxygen vacancies of $\mathrm{ZnO} .^{38,39}$ In the case of the $\mathrm{ZnO}: \mathrm{Co@ZIF-8} \mathrm{core-shell}$ NRs, the BE is highly quenched, while the defect band of the oxygen vacancies still shows luminescence. The decrease in the $\mathrm{PL}$ intensity of the $\mathrm{BE}$ combined with the decrease in the decay time for TA bleaching in the presence of the Co doping and the ZIF-8 suggests to two distinct and simultaneous influences: the availability of multiple nonradiative recombination pathways and carrier trapping by the increased surface defects in the presence of doping and the ZIF- 8 . Figure $4 \mathrm{~h}$ shows stability measurements performed in the four photoanodes to assess the influence of the shell on the performance stability over time of the NRs. The MOF shell passivates the surface of the NRs against photocorrosion, which doubles the average lifetime compared to the pristine NRs. Cobalt doping also slightly enhances the stability over time, although its influence is minor compared to that of the shell. Further studies of the influence of different shells on the photoelectrochemical performance are shown in Figure S11.

A schematic band structure and functional mechanism diagrams are illustrated in Figure 5. Under illumination, electron-hole pairs are generated at both the Co-doped $\mathrm{ZnO}$ core and the ZIF-8 shell ${ }^{40}$ (see Figure S12). In the Co-doped $\mathrm{ZnO}$ core, electrons have two excitation possibilities, i.e., either to the conduction band or to the $\mathrm{sp}-\mathrm{d}$ related localized states introduced by the Co-doping. ${ }^{35,36,41}$ Meanwhile, holes can travel only to the ZIF- 8 shell or recombine. Nevertheless, for the hole transfer to the ZIF- 8 shell, the energy difference between the valence band of the Co-doped $\mathrm{ZnO}$ and $\mathrm{ZIF}-8$ should be small. The Mott-Schottky measurements and the bandgap show that these two levels are $0.25 \mathrm{eV}$ apart, ${ }^{40}$ which is a sufficiently small enough energy barrier for the photogenerated holes to be injected from the core into the shell. Accordingly, the shell acts as a hole transfer layer between the $\mathrm{ZnO}$ core and the electrolyte, enhancing the electron-hole charge separation. In addition, the positions of the conduction band of the $\mathrm{ZnO}$ core and the ZIF- 8 are such that they enable the electron transfer from the shell to the core and from the $\mathrm{ZnO}$ core to the counter electrode through the ITO substrate. This electron and hole transfer from and to the ZIF-8 shell is provided by the chemical structure of the imidazole linker. In ZIF-8, the valence band is localized in the $\mathrm{N}$ atoms from the two amine groups, while the conduction band is formed by the empty orbitals of $\mathrm{Zn} .{ }^{42}$ Thus, any photogenerated electrons will travel to the $\mathrm{Zn}$ cluster and from there to the NRs by a process known as ligand-to-cluster charge transfer ${ }^{43}$ (LCCT). Meanwhile, holes will be injected through the amine groups into water molecules adsorbed to shell hydrogens, initiating the 


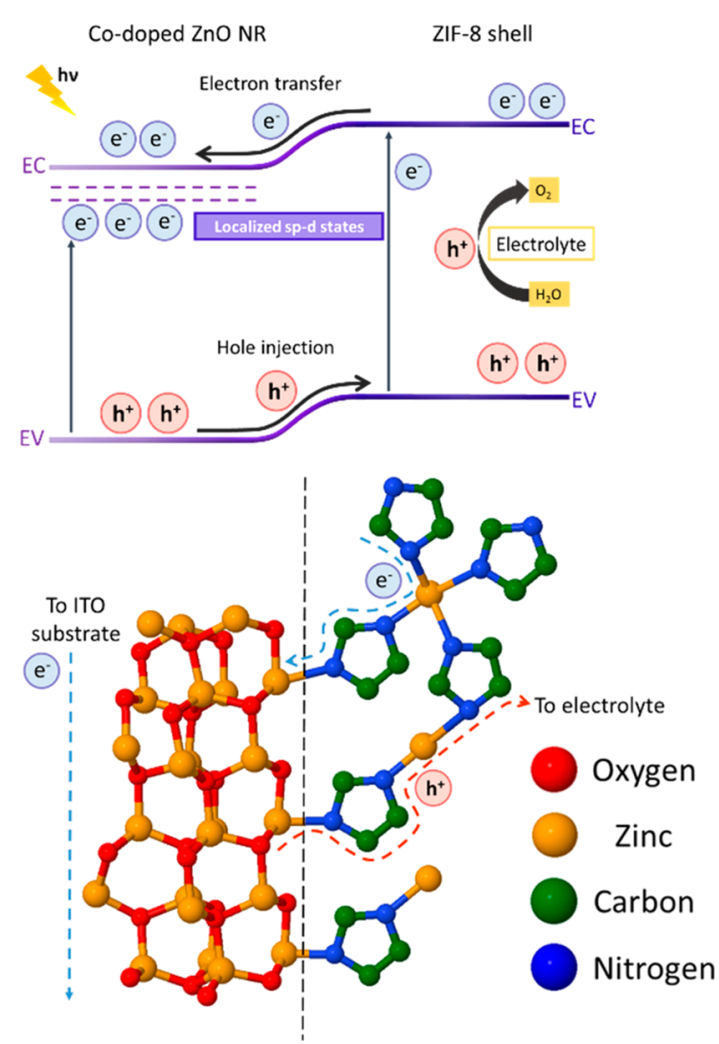

Figure 5. (top) Schematic of the proposed electronic band diagram showing the interface between the Co-doped core and the ZIF-8 shell and the flow of charge carriers. In this schematic, EV represents the valence band, EC the conduction band, $\mathrm{e}^{-}$electrons, and $\mathrm{h}^{+}$holes. (bottom) Schematic of the molecular structure of the ZnO:Co@ZIF8 , showing the $\mathrm{ZnO}$ wurtzite lattice on the left, coordinated to the ZIF-8 shell on the right. For clarity, only a tetrahedral unit of ZIF-8 represented in two dimensions is shown, and no Co is shown in the $\mathrm{ZnO}$ : $\mathrm{Co}$ core lattice. The flow of charges through the Co-doped $\mathrm{ZnO}$ core and the ZIF- 8 is shown to illustrate the overall working mechanism of the ZnO:Co@ZIF-8 structure as a photoanode.

water-splitting process as represented by the black and yellow schematic on the right side of the electronic band diagram of Figure 5.

\section{CONCLUSIONS}

In conclusion, we have developed a simple, effective route for the growth of highly efficient core-shell ZnO:Co@ZIF-8 doped NRs as water-splitting photoanodes. A fast, facile growth method for the ZIF-8 shell by spin-coating is also demonstrated. The design of this semiconductor system doubles the current density obtained from pristine $\mathrm{ZnO}$ NRs while yielding an unprecedented IPCE of $75 \%$ at $350 \mathrm{~nm}$ because of improved light absorption and charge separation and transfer provided by the synergetic Co-doping and the ZIF-8 shell combination. Furthermore, the shell acts as a passivating coating on the NRs against photocorrosion, enhancing by a factor of 2 the functional lifetime of the core-shell photoanodes. The proposed electronic structure of the ZnO:Co@ZIF-8 NRs and the charge transfer mechanism show that this synergetic integration of doping and passivation opens a new pathway for the development of low-cost, green, water-based, scalable, and highly efficient semiconductor-based photoanodes that could be key in a future hydrogen-based economy, while also offering the potential for other applications such as chemical sensing.

\section{EXPERIMENTAL SECTION}

$\mathrm{ZnO}$ Nanorod Growth. The $\mathrm{ZnO}$ nanorods (NRs) were grown by seeded-mediated chemical bath deposition (CBD). First, indium tin oxide (ITO) on glass substrates of $2 \mathrm{~cm}$ by $0.8 \mathrm{~cm}$ were thoroughly cleaned with isopropanol and then treated with ozone for $30 \mathrm{~min}$ to enable favorable adhesion of the $\mathrm{ZnO}$ seed layer to the substrate. A $\mathrm{ZnCl}_{2}$ solution $(225 \mathrm{mg})$ in 2-methoxyethanol $(10 \mathrm{~mL})$ was prepared and spin-coated on the cleaned substrate at $5000 \mathrm{rpm}$ for $1 \mathrm{~min}$. For the NR growth, an equimolar solution containing 25 $\mathrm{mM}$ concentration of zinc nitrate hexahydrate and hexamethylenetetramine in deionized water (DI water, $200 \mathrm{~mL}$ ) was prepared by magnetic stirring until a clear solution was obtained. The seeded ITO substrates were then submerged facedown in the solution supported by a custom-made holder and introduced into an oven at $90{ }^{\circ} \mathrm{C}$ for 6 h. After growth, the samples were cleaned under a DI water stream and blow-dried with nitrogen gas. For the electrochemical measurements, a $0.8 \mathrm{~cm}$ by $0.5 \mathrm{~cm}$ region of the ITO substrates was covered with Kapton tape during NR growth so the ITO remained untouched and could be used directly as electrical contact.

Doping of the ZnO NRs. Cobalt doping was introduced in the NRs by adding the desired concentration of cobalt nitrate hexahydrate to the growth solution. The cobalt source at desired concentrations was dissolved in the water solution at the same time as the zinc source and hexamethylenetetramine. Resulting concentrations are expressed as molar $\mathrm{Co} / \mathrm{Zn}$ percentages. In the paper the $1 \%$ Co-doped sample was discussed.

Growth of the Core-Shell Structure. The ZIF-8 shell was grown by spin-coating and surface conversion of the NRs on a hot plate. First, the desired concentration of the organic linker, 2methylimidazole, was dissolved in methanol $(10 \mathrm{~mL})$ with magnetic stirring. In this work, a $1 \mathrm{M}$ concentration was used. The imidazole solution was then spin-coated onto the NRs at $5000 \mathrm{rpm}$ for $30 \mathrm{~s}$. In a typical spin-coating process, 3-5 droplets of the imidazole solution were sufficient to create a continuous wetting layer on the surface of the as-grown NRs. After spin-coating, a two-step growth was performed on a hot plate. First, a pregrowth evaporation step at 65 ${ }^{\circ} \mathrm{C}$ for $5 \mathrm{~min}$ was performed to remove all the remaining methanol. Then, the surface conversion reaction of the ZnO NRs into ZIF-8 was performed at $160^{\circ} \mathrm{C}$ for $30 \mathrm{~min}$. The samples were then rinsed with methanol and blow-dried with nitrogen. The shell was tuned by varying the concentration of 2-methylimidazole in methanol, thus termed shell concentration.

Microscopy Analyses. Field emission scanning electron microscopy (FESEM) micrographs of the NRs were obtained by using a FEI Helios Nanolab 600 at $10 \mathrm{kV}$. Transmission electron microscopy (TEM) imaging and energy dispersive X-ray spectroscopy (EDX) were performed in a JEOL 2100F FEG at $200 \mathrm{kV}$ equipped with an Oxford INCAx-sight $\mathrm{Si}(\mathrm{Li})$ detector with a $50 \mathrm{~mm}^{2}$ area detector at $25^{\circ}$ take-off angle.

Photoelectrochemical Characterization. Water-splitting experiments were performed in a three-electrode photoelectrochemical cell, in which films of the materials prepared, deposited on FTO substrates, were used as photoanodes. The photoanode corresponds to the oxidation reaction, whereby the related current and IPCE values refer to the oxygen production. From this the hydrogen production (taking actually place at the Pt counter electrode) can be evaluated. An $\mathrm{HgO} / \mathrm{Hg}$ electrode was used as the reference electrode for the measurements. The photoelectrochemical measurements were performed with an Autolab PGSTAT302N potentiostat using a LOT ORIEL solar simulator LS0106 as light source, generating AM 1.5G light from a Xe Arc lamp $(150 \mathrm{~W})$ using $300 \mathrm{~mW} \mathrm{~cm}^{-2}$. For the incident photon to current efficiency (IPCE) measurements, a LOT Oriel MSH-300 monochromator was used.

Optical Characterization. UV-vis spectroscopy measurements were performed with a Shimadzu UV-1800 spectrophotometer. For the fast transient absorption spectroscopy measurements (FTAS), 
samples were measured in pump-probe configuration. As the pump, the output of an optical parametric amplifier (OPA) was used, while the white light continuum (WLC) generated in a femtosecond transient absorption spectrometer of IB Photonics (FemtoFrame II) was used as probe. The white light supercontinuum is generated for the visible region with $800 \mathrm{~nm}$ passing through $\mathrm{CaF}_{2}$ crystal. The pump-probe response was studied in the visible region of the spectrum. In this work, we used a pump of $275 \mathrm{~nm}(4.51 \mathrm{eV})$ and an excitation intensity of $260 \mu \mathrm{J} \mathrm{cm}^{-2}$. The instrument response function was measured to be $\approx 50 \mathrm{fs}$. The quantity measured is the difference in absorbance $(\Delta A)$ of the probe transmitted through the excited sample and that transmitted through the unperturbed sample as a function of the delay time between pump and probe and probe wavelength (energy). Photoluminescence spectroscopy was measured in the backscattering configuration by using the same excitation as the pump extracted from the OPA for the FTAS measurements.

\section{ASSOCIATED CONTENT}

\section{SI Supporting Information}

The Supporting Information is available free of charge at https://pubs.acs.org/doi/10.1021/acsanm.0c01325.

A brief description of the literature comparison; XRD and XPS analyses of doped core-shell nanowires; further supplementary microscopy analyses of the doped core-shell nanostructures; additional photoelectrochemical characterization of the pristine and core-shell nanomaterials (PDF)

\section{AUTHOR INFORMATION}

\section{Corresponding Authors}

Alejandro Galán-González - Department of Engineering and Department of Physics, Durham University, Durham DH1 3LE, U.K.; @ orcid.org/0000-0002-8217-7445;

Email: alejandro.galan@durham.ac.uk

Del Atkinson - Department of Physics, Durham University, Durham DH1 3LE, U.K.; Email: del.atkinson@durham.ac.uk

\section{Authors}

Aswathi K. Sivan - Istituto per la Microelettronica e i Microsistemi (IMM)-CNR, I-00133 Rome, Italy,

Javier Hernández-Ferrer - Instituto de Carboquimica (ICBCSIC), 50018 Zaragoza, Spain; 이잉.org/0000-00026586-6935

Leon Bowen - Department of Physics, Durham University, Durham DH1 3LE, U.K.

Lorenzo Di Mario - Istituto di Struttura della Materia (ISMCNR), I-00133 Rome, Italy

Faustino Martelli - Istituto per la Microelettronica e $i$ Microsistemi (IMM)-CNR, I-00133 Rome, Italy; 이이. ord.org/ 0000-0002-4496-4165

Ana M. Benito - Instituto de Carboquímica (ICB-CSIC), 50018 Zaragoza, Spain; 이이이.org/0000-0002-8654-7386

Wolfgang K. Maser - Instituto de Carboquimica (ICB-CSIC), 50018 Zaragoza, Spain; (1) orcid.org/0000-0003-4253-0758

Mujeeb Ullah Chaudhry - Department of Engineering, Durham University, Durham DH1 3LE, U.K.; (1) orcid.org/ 0000-0002-6149-3457

Andrew Gallant - Department of Engineering, Durham University, Durham DH1 3LE, U.K.

Dagou A. Zeze - Department of Engineering, Durham University, Durham DH1 3LE, U.K.; ITMO University, St. Petersburg 197101, Russia

Complete contact information is available at: https://pubs.acs.org/10.1021/acsanm.0c01325

\section{Author Contributions}

A.G.-G. and D.A. jointly conceived the project and wrote the manuscript. A.G.-G. performed the growth of all the nanostructures and the microscopy analyses with the help of L.B. Optical FTAS characterization was performed by A.K.S., who also performed the analyses of the results. L.D.M. provided technical support for the FTAS measurements. J.H.F. performed the photoelectrochemical studies and analyzed the data jointly with A.G.-G. All authors discussed the results and contributed to the manuscript.

\section{Notes}

The authors declare no competing financial interest.

\section{ACKNOWLEDGMENTS}

A.G.-G., A.K.S., F.M., A.G., D.A.Z., and D.A. acknowledge funding received from the European Union's Horizon 2020 research and innovation programme under the Marie Skłodowska-Curie Grant Agreement No 722176. W.K.M., A.M.B., and J.H.F. acknowledge financial support from Spanish Ministry MINEICO under Project ENE2016-79282-C5-1-R (AEI/UE/FEDER) and the Gobierno de Aragón (Grupo Reconocido DGA T03_17R, FEDER/UE y DGA T03_20R).

\section{REFERENCES}

(1) Tembhurne, S.; Nandjou, F.; Haussener, S. A thermally synergistic photo-electrochemical hydrogen generator operating under concentrated solar irradiation. Nature Energy 2019, 4, 399-407.

(2) Fabbri, E.; Habereder, A.; Waltar, K.; Kötz, R.; Schmidt, T. J. Developments and perspectives of oxide-based catalysts for the oxygen evolution reaction. Catal. Sci. Technol. 2014, 4, 3800-3821.

(3) Grätzel, M. Photoelectrochemical cells. Nature 2001, 414, 338344.

(4) Walter, M. G.; Warren, E. L.; McKone, J. R.; Boettcher, S. W.; Mi, Q.; Santori, E. A.; Lewis, N. S. Solar water splitting cells. Chem. Rev. 2010, 110, 6446-73.

(5) Staffell, I.; Scamman, D.; Velazquez Abad, A.; Balcombe, P.; Dodds, P. E.; Ekins, P.; Shah, N.; Ward, K. R. The role of hydrogen and fuel cells in the global energy system. Energy Environ. Sci. 2019, $12,463-491$.

(6) Pinaud, B. A.; Benck, J. D.; Seitz, L. C.; Forman, A. J.; Chen, Z.; Deutsch, T. G.; James, B. D.; Baum, K. N.; Baum, G. N.; Ardo, S. Wang, H.; Miller, E.; Jaramillo, T. F. Technical and economic feasibility of centralized facilities for solar hydrogen production via photocatalysis and photoelectrochemistry. Energy Environ. Sci. 2013, 6, 1983.

(7) Shaner, M. R.; Atwater, H. A.; Lewis, N. S.; McFarland, E. W. A comparative technoeconomic analysis of renewable hydrogen production using solar energy. Energy Environ. Sci. 2016, 9, 23542371.

(8) Pagliaro, M.; Konstandopoulos, A. G.; Ciriminna, R.; Palmisano, G. Fuels of the future. Energy Environ. Sci. 2010, 3, 253.

(9) Tollefson, J. Hydrogen vehicles: fuel of the future. Nature 2010, $464,1262-1264$.

(10) Hanley, E. S.; Deane, J.; Gallachóir, B. Ó. The role of hydrogen in low carbon energy futures-A review of existing perspectives. Renewable Sustainable Energy Rev. 2018, 82, 3027-3045.

(11) Pivovar, B. Catalysts for fuel cell transportation and hydrogen related uses. Nature Catalysis 2019, 2, 562-565.

(12) Yang, H.; Bright, J.; Kasani, S.; Zheng, P.; Musho, T.; Chen, B.; Huang, L.; Wu, N. Metal-organic framework coated titanium dioxide nanorod array $\mathrm{p}-\mathrm{n}$ heterojunction photoanode for solar watersplitting. Nano Res. 2019, 12, 643-650.

(13) Tang, P.-Y.; Han, L.-J.; Hegner, F. S.; Paciok, P.; Biset-Peiró, M.; Du, H.-C.; Wei, X.-K.; Jin, L.; Xie, H.-B.; Shi, Q.; Andreu, T.; Lira-Cantú, M.; Heggen, M.; Dunin-Borkowski, R. E.; López, N.; Galán-Mascarós, J. R.; Morante, J. R.; Arbiol, J. Boosting Photo- 
electrochemical Water Oxidation of Hematite in Acidic Electrolytes by Surface State Modification. Adv. Energy Mater. 2019, 9, 1901836.

(14) Wolcott, A.; Smith, W. A.; Kuykendall, T. R.; Zhao, Y.; Zhang, J. Z. Photoelectrochemical Study of Nanostructured ZnO Thin Films for Hydrogen Generation from Water Splitting. Adv. Funct. Mater. 2009, 19, 1849-1856.

(15) Wang, M.; Ren, F.; Zhou, J.; Cai, G.; Cai, L.; Hu, Y.; Wang, D.; Liu, Y.; Guo, L.; Shen, S. N Doping to ZnO Nanorods for Photoelectrochemical Water Splitting under Visible Light: Engineered Impurity Distribution and Terraced Band Structure. Sci. Rep. 2015, 5, 12925.

(16) Kegel, J.; Povey, I. M.; Pemble, M. E. Zinc oxide for solar water splitting A brief review of the material's challenges and associated opportunities. Nano Energy 2018, 54, 409-428.

(17) Kuo, D.-Y.; Eom, C. J.; Kawasaki, J. K.; Petretto, G.; Nelson, J. N.; Hautier, G.; Crumlin, E. J.; Shen, K. M.; Schlom, D. G.; Suntivich, J. Influence of Strain on the Surface-Oxygen Interaction and the Oxygen Evolution Reaction of SrIrO3. J. Phys. Chem. C 2018, 122, 4359-4364.

(18) Janotti, A.; Van de Walle, C. G. Fundamentals of Zinc Oxide as a Semiconductor. Rep. Prog. Phys. 2009, 72, 126501.

(19) Patel, P. P.; Hanumantha, P. J.; Velikokhatnyi, O. I.; Datta, M. K.; Hong, D.; Gattu, B.; Poston, J. A.; Manivannan, A.; Kumta, P. N. Nitrogen and cobalt co-doped zinc oxide nanowires - Viable photoanodes for hydrogen generation via photoelectrochemical water splitting. J. Power Sources 2015, 299, 11-24.

(20) Wang, G.; Yang, X.; Qian, F.; Zhang, J. Z.; Li, Y. Double-sided $\mathrm{CdS}$ and $\mathrm{CdSe}$ quantum dot co-sensitized $\mathrm{ZnO}$ nanowire arrays for photoelectrochemical hydrogen generation. Nano Lett. 2010, 10, 1088-92.

(21) Ahn, K.-S.; Yan, Y.; Shet, S.; Deutsch, T.; Turner, J.; Al-Jassim, $\mathrm{M}$. Enhanced photoelectrochemical responses of $\mathrm{ZnO}$ films through Ga and N codoping. Appl. Phys. Lett. 2007, 91, 231909.

(22) Roger, I.; Shipman, M. A.; Symes, M. D. Earth-abundant catalysts for electrochemical and photoelectrochemical water splitting. Nat. Rev. Chem. 2017, 1, No. 0003.

(23) Kaphle, A.; Hari, P. Variation of index of refraction in cobalt doped $\mathrm{ZnO}$ nanostructures. J. Appl. Phys. 2017, 122, 165304.

(24) Furukawa, H.; Cordova, K. E.; O’Keeffe, M.; Yaghi, O. M. The chemistry and applications of metal-organic frameworks. Science 2013, $341,1230444$.

(25) Yaghi, O. M.; O’Keeffe, M.; Ockwig, N. W.; Chae, H. K.; Eddaoudi, M.; Kim, J. Reticular synthesis and the design of new materials. Nature 2003, 423, 705-714.

(26) Park, K. S.; Ni, Z.; Côté, A. P.; Choi, J. Y.; Huang, R.; UribeRomo, F. J.; Chae, H. K.; O’Keeffe, M.; Yaghi, O. M. Exceptional chemical and thermal stability of zeolitic imidazolate frameworks. Proc. Natl. Acad. Sci. U. S. A. 2006, 103, 10186-10191.

(27) Chen, B.; Yang, Z.; Zhu, Y.; Xia, Y. Zeolitic imidazolate framework materials. Recent progress in synthesis and applications. J. Mater. Chem. A 2014, 2, 16811-16831.

(28) Lu, G.; Hupp, J. T. Metal-Organic Frameworks as Sensors. A ZIF-8 Based Fabry-Pérot Device as a Selective Sensor for Chemical Vapors and Gases. J. Am. Chem. Soc. 2010, 132, 7832-7833.

(29) Zhan, W.-W.; Kuang, Q.; Zhou, J.-Z.; Kong, X.-J.; Xie, Z.-X.; Zheng, L.-S. Semiconductor@Metal-Organic Framework Core-Shell Heterostructures. A Case of ZnO@ZIF-8 Nanorods with Selective Photoelectrochemical Response. J. Am. Chem. Soc. 2013, 135, 19261933.

(30) Li, X.; Liu, S.; Fan, K.; Liu, Z.; Song, B.; Yu, J. MOF-Based Transparent Passivation Layer Modified ZnO Nanorod Arrays for Enhanced Photo-Electrochemical Water Splitting. Adv. Energy Mater. 2018, 8, 1800101.

(31) Jia, G.; Liu, L.; Zhang, L.; Zhang, D.; Wang, Y.; Cui, X.; Zheng, W. 1D alignment of $\mathrm{ZnO@ZIF8-67} \mathrm{nanorod} \mathrm{arrays} \mathrm{for} \mathrm{visible-light-}$ driven photoelectrochemical water splitting. Appl. Surf. Sci. 2018, 448, 254-260.
(32) Greene, L. E.; Law, M.; Tan, D. H.; Montano, M.; Goldberger, J.; Somorjai, G.; Yang, P. General Route to Vertical ZnO Nanowire Arrays Using Textured ZnO Seeds. Nano Lett. 2005, 5, 1231-1236.

(33) Galan-Gonzalez, A.; Gallant, A.; Zeze, D. A.; Atkinson, D. Controlling the growth of single crystal $\mathrm{ZnO}$ nanowires by tuning the atomic layer deposition parameters of the $\mathrm{ZnO}$ seed layer. Nanotechnology 2019, 30, 305602.

(34) Wilson, R. H. Observation and Analysis of Surface States on $\mathrm{TiO}_{2}$ Electrodes in Aqueous Electrolytes. J. Electrochem. Soc. 1980, 127, 228-234.

(35) Kegel, J.; Povey, I. M.; Pemble, M. E. Zinc oxide for solar water splitting: A brief review of the material's challenges and associated opportunities. Nano Energy 2018, 54, 409-428.

(36) May, J. W.; Ma, J.; Badaeva, E.; Li, X. Effect of Excited-State Structural Relaxation on Midgap Excitations in Co2+-Doped $\mathrm{ZnO}$ Quantum Dots. J. Phys. Chem. C 2014, 118, 13152-13156.

(37) Lu, Y.; Lin, Y.; Wang, D.; Wang, L.; Xie, T.; Jiang, T. A high performance cobalt-doped $\mathrm{ZnO}$ visible light photocatalyst and its photogenerated charge transfer properties. Nano Res. 2011, 4, 11441152.

(38) Janotti, A.; Van de Walle, C. G. Oxygen vacancies in ZnO. Appl. Phys. Lett. 2005, 87, 122102.

(39) McCluskey, M. D.; Jokela, S. J. Defects in ZnO. J. Appl. Phys. 2009, 106, No. 071101.

(40) Li, P.; Li, J.; Feng, X.; Li, J.; Hao, Y.; Zhang, J.; Wang, H.; Yin, A.; Zhou, J.; Ma, X.; Wang, B. Metal-organic frameworks with photocatalytic bactericidal activity for integrated air cleaning. Nat. Commun. 2019, 10, 2177.

(41) Sutka, A.; Käämbre, T.; Pärna, R.; Juhnevica, I.; Maiorov, M.; Joost, U.; Kisand, V. Co doped $\mathrm{ZnO}$ nanowires as visible light photocatalysts. Solid State Sci. 2016, 56, 54-62.

(42) Chin, M.; Cisneros, C.; Araiza, S. M.; Vargas, K. M.; Ishihara, K. M.; Tian, F. Rhodamine B degradation by nanosized zeolitic imidazolate framework-8 (ZIF-8). RSC Adv. 2018, 8, 26987-26997.

(43) Grau-Crespo, R.; Aziz, A.; Collins, A. W.; Crespo-Otero, R.; Hernandez, N. C.; Rodriguez-Albelo, L. M.; Ruiz-Salvador, A. R.; Calero, S.; Hamad, S. Modelling a Linker Mix-and-Match Approach for Controlling the Optical Excitation Gaps and Band Alignment of Zeolitic Imidazolate Frameworks. Angew. Chem., Int. Ed. 2016, 55, 16012-16016. 